\title{
Kinematic Parameter Identification of a Binocular Head Using Stereo Measurements of Single Calibration Point
}

\author{
Sheng-Wen Shih ${ }^{2}$, Yi-Ping Hung ${ }^{1]}$, Wei-Song Lin ${ }^{2}$ \\ [1] Institute of Information Science, Academia Sinica, Nankang, Taipei, Taiwan. \\ ${ }^{22}$ Institute of Electrical Engineering, National Taiwan University, Taipei, Taiwan. \\ e-mail: hung@iis.sinica.edu.tw
}

\begin{abstract}
This paper proposes a new closed-form solution for identifying the kinematic parameters of an active binocular head by using a single calibration point. This method is based on the complete and parametrically continuous $(C P C)$ kinematic model, and can be applied to any kind of kinematic parameter identification problems with or without multiple end-effectors, providing that the links are rigid, the joints are either revolute or prismatic and no closed-loop kinematic chain is included. As a practical example, this paper focuses on the calibration of a binocular head having four revolute joints and two prismatic joints. Simulation and real experiments have shown that the proposed method of using point measurements can achieve much higher accuracy than that of using pose measurements.
\end{abstract}

\section{Introduction}

Many computer vision problems that are ill-posed, nonlinear or unstable for a passive observer become well-posed, linear or stable for an active observer[1]. Being able to acquire information actively, the active vision system has more potential applications than a passive one has. In an active stereo vision system, the cameras are able to preform functions such as gazing, panning and tilting. To perform experiments on active vision, we have built a binocular head (referred to as the IIS-Head). This IIS-Head has four revolute joints and two prismatic joints, as shown in Fig. 1. The two joints on top of the IIS-Head are for camera vergence or gazing (referred to as joint $5 L$ and joint $5 R$ ). The next two joints below them are for tilting and panning the stereo cameras (referred to as joint 4 and joint 3 ). All of the above four joints are revolute, and are mounted on an X-Y table which is composed of two prismatic joints (referred to as joint 2 and joint 1). Our goal is to calibrate its kinematic parameters for controlling the orientations and positions of the stereo cameras. Since the IIS-Head is built with off-the-shelf components, its kinematic parameters are unknown. Therefore, we cannot directly apply those existing iterative methods requiring nominal model (initial values), e.g, [3][5][13].
For practical reasons stated in [9], we chose to use a twostage approach (including a head calibration stage and a head/eye calibration stage) for calibrating the binocular head. This paper will focus on the first stage of the calibration, i.e, the head calibration (however, the method can be applied to the kinematic calibration of general robots). At the head calibration stage, the two cameras on the IIS-Head are replaced by two small end-effector calibration plates. The positions of the calibration plates, or equivalently, of the end-effectors, can be estimated by using the stereo vision measurement system. The acquired positions of the calibration plates are then used to calibrate the kinematic parameters.

Due to the lack of a nominal kinematic model for our IIS head, only those calibration techniques providing closedform solutions are considered. Both the methods proposed by Zhuang[14] and Shih[8] give direct solution to the CPC kinematic parameters[13], by using the end effector's poses measurements. Lenz and Tsai[4] and Young et. al.[12] estimate the joint axis from the measurements of the end-effector's poses. However, in practice, it is more difficult to accurately estimate the end-effector's pose than to estimate the

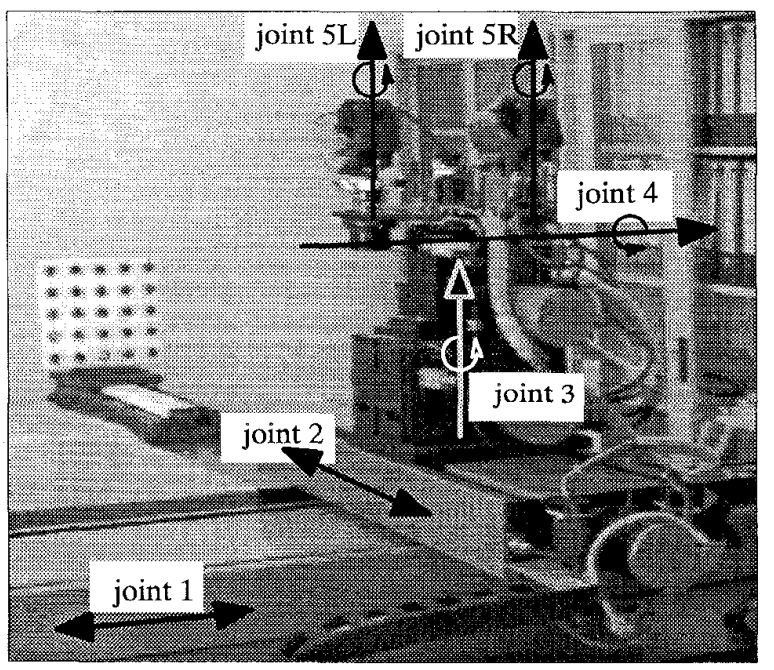

Fig. 1. A picture of the IIS-Head 
3D coordinates of a single calibration point on the end-effector. Stone[10] and Sklar[11] estimate the joint axis from the measurements of the 3D coordinates of a calibration point attached to the robot arm, while moving each robot joint one at a time. When moving a revolute joint, the trajectory of the calibration point forms a $3 \mathrm{D}$ circle. First, a plane is fitted to the measured calibration points by least-square technique. The resulted plane normal defines the orientation of the revolute joint axis. The measured calibration points are then projected along the plane normal onto the plane. A $2 \mathrm{D}$ circle is then fitted, again by least-square technique, to find its center which defines the location of the joint axis. In this approach, the orientation and location of the joint axis are separately estimated without using the information contained in the amount of rotation angles.

In this paper, we shall present a closed-form solution based on the CPC kinematic model using single calibration point. The advantages of using the $\mathrm{CPC}$ kinematic model are described in [13] and [14].

This paper is organized as follows. The kinematic calibration problem is formulated in section II. The new calibration method is described in section III. Experimental results are shown in section IV. Conclusions are given in section $\mathrm{V}$.

\section{Problem formulation}

The CPC kinematic model we use for a revolute or prismatic joint is as follows (see [13] and [9] for more details).

$$
\begin{aligned}
& { }^{i} \boldsymbol{T}_{i+1}={ }^{i} \boldsymbol{T}_{i^{\prime}}{ }^{\prime} \boldsymbol{T}_{i+1}, \\
& \text { where } \quad{ }^{i} \boldsymbol{T}_{\boldsymbol{i}^{\prime}} \equiv \boldsymbol{Q}_{i} \text {, } \\
& { }^{i^{\prime}} \boldsymbol{T}_{i+1} \equiv \boldsymbol{V}_{i} \text {, } \\
& Q_{i}= \begin{cases}\operatorname{Rot}_{z}\left(q_{i}\right), & \text { for revolute joint, } \\
\operatorname{Trans}\left(\left[\begin{array}{lll}
0 & 0 & q_{i}
\end{array}\right]^{T}\right), & \text { for prismatic joint, }\end{cases}
\end{aligned}
$$

$$
\begin{aligned}
& \boldsymbol{R o t}_{z}(\theta) \equiv\left[\begin{array}{cccc}
\cos (\theta) & -\sin (\theta) & 0 & 0 \\
\sin (\theta) & \cos (\theta) & 0 & 0 \\
0 & 0 & 1 & 0 \\
0 & 0 & 0 & 1
\end{array}\right] \\
& \operatorname{Trans}\left(\left[\begin{array}{lll}
x & y & z
\end{array}\right]^{T}\right) \equiv\left[\begin{array}{llll}
1 & 0 & 0 & x \\
0 & 1 & 0 & y \\
0 & 0 & 1 & z \\
0 & 0 & 0 & 1
\end{array}\right] \\
& q_{i}=s_{i} q_{i}^{\prime}, s_{i} \in\{+1,-1\} \text {, } \\
& q^{\prime}{ }_{i} \text { is the } i \text { th joint value, } \\
& V_{i}=\boldsymbol{R}_{i} \operatorname{Rot}_{z}\left(\beta_{i}\right) \operatorname{Trans}\left(\left[\begin{array}{lll}
l_{i, x} & l_{i, y} & l_{i, z}
\end{array}\right]^{T}\right) \text {, } \\
& \text { and } \boldsymbol{R}_{i}=\left[\begin{array}{cccc}
1-\frac{b_{i, x}^{2}}{1+b_{i, z}} & \frac{-b_{i, x} b_{i, y}}{1+b_{i, z}} & b_{i, x} & 0 \\
\frac{-b_{i, x} b_{i, y}}{1+b_{i, z}} & 1-\frac{b_{i, y}^{2}}{1+b_{i, z}} & b_{i, y} & 0 \\
-b_{i, x} & -b_{i, y} & b_{i, z} & 0 \\
0 & 0 & 0 & 1
\end{array}\right] \text {. }
\end{aligned}
$$

Notice that for convenience we have introduced an intermediate coordinate system between $i$ th and $(i+l)$ st frame, i.e., the $i^{\prime}$ frame in equation (1). From the frame $i$ to the frame $i^{\prime}$, it is either a rotational or a translational motion matrix depending on the joint type, and from the frame $i^{\prime}$ to the frame $i+1$, it is a fixed link matrix (referred to as "shape" matrix in [13]) as defined in equation (6).

Different coordinate systems associated with different joints of the IIS-Head are shown in Fig. 2 The coordinate frames $\{6 L\}$ and $\{6 R\}$ are the end-effector coordinate systems. As shown in Fig. 3, we have the following relations:

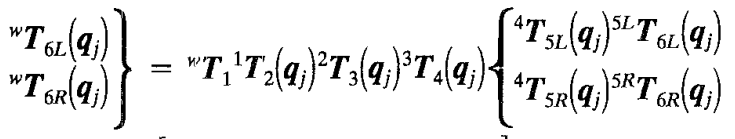

where $\boldsymbol{q}_{j}=\left[\begin{array}{llllll}q_{1 j} & q_{2 j} & q_{3 j} & q_{4 j} & q_{5 R j} & q_{5 L j}\end{array}\right]$ denotes the joint values for the $j$ th robot configuration, ${ }^{i} \boldsymbol{T}_{k}$ denotes the transformation matrix from frame $i$ to frame $k$. For convenience,

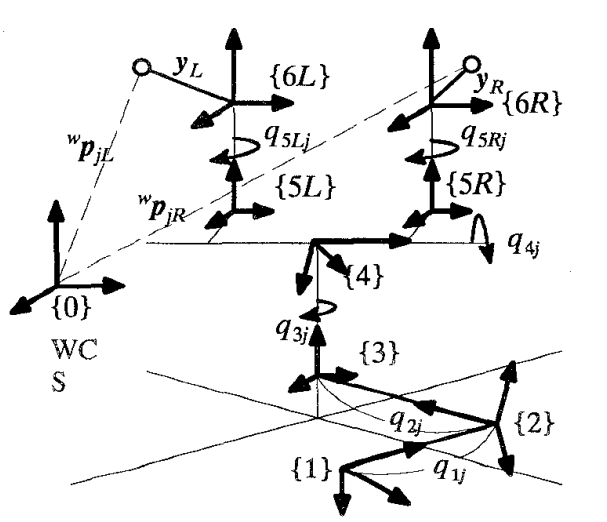

Fig. 2. The skeleton diagram of the IIS-Head.

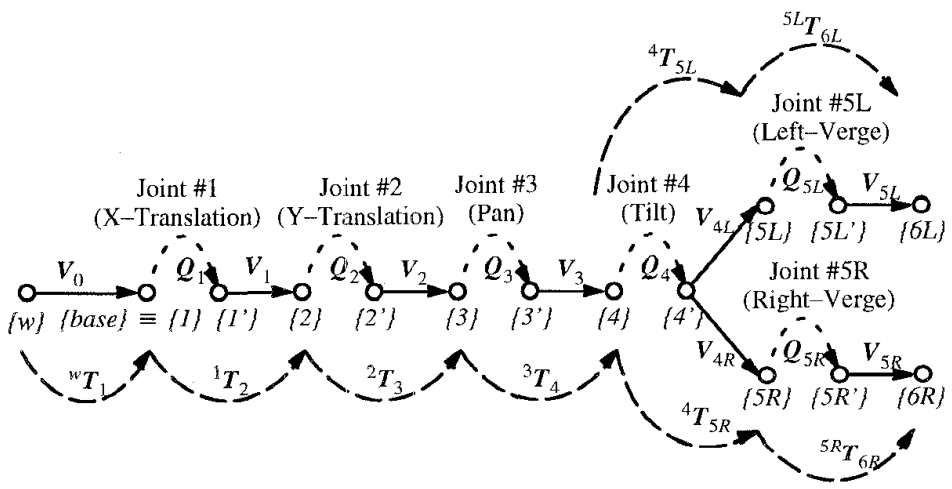

Fig. 3. Kinematic Reference Frames of the IIS-Head 
each of the transformation matrix is written as a function of the joint value vector, $\boldsymbol{q}_{j}$, instead of $\boldsymbol{q}_{i j}$.

Suppose that the fixed coordinates of the calibration points mounted on the left and right end-effectors are $\boldsymbol{y}_{L}$ and $y_{R}$ with respect to LECS (left end-effector coordinate system) and RECS (right end-effector coordinate system), respectively. Let ${ }^{w} \boldsymbol{p}_{j L}$ and ${ }^{w} \boldsymbol{p}_{j R}$ denote the coordinates of the calibration points, corresponding to the $j$ th robot configuration $\boldsymbol{q}_{j}$, measured in the WCS. Transforming the coordinates of the calibration points, $\boldsymbol{y}_{L}$ and $\boldsymbol{y}_{\boldsymbol{R}}$, from LECS and RECS to the WCS, we have

$$
\text { and } \quad \begin{aligned}
{ }^{w} \tilde{p}_{j L} & ={ }^{w} T_{6 L}\left(q_{j}\right) \tilde{y}_{L}, \\
{ }^{w} \tilde{p}_{j R} & ={ }^{w} \boldsymbol{T}_{6 R}\left(\boldsymbol{q}_{j}\right) \tilde{y}_{R},
\end{aligned}
$$

where ${ }^{w} \tilde{\boldsymbol{p}}_{j L},{ }^{w} \tilde{\boldsymbol{p}}_{j R}, \tilde{\boldsymbol{y}}_{L}$ and $\tilde{\boldsymbol{y}}_{R}$ are the $3 \mathrm{D}$ homogeneous coordinates of ${ }^{\boldsymbol{w}} \boldsymbol{p}_{j L},{ }^{\boldsymbol{w}} \boldsymbol{p}_{j R}, \boldsymbol{y}_{L}$ and $\boldsymbol{y}_{R}$, respectively.

With our new method, each joint is calibrated from the base toward the end-effectors. Consider Fig. 4. Without loss of generality, we assume that the kinematic parameters of the joints from the base to the $i$ th joint have been known when calibrating the $i$ th link matrix, i.e., $V_{i}$. Only those joints with known kinematic parameters plus the $(i+l)$ st joint are permitted to be moved. For example, when calibrating $V_{4 L}$, joints $1,2,3,4$ and $5 L$ are allowed to be moved. From equations (8), (9) and (10), we have

$$
\begin{aligned}
& { }^{i} \tilde{p}_{j L}={ }^{i} \boldsymbol{T}_{i+1}{ }^{i+1} \boldsymbol{T}_{(i+1)^{\prime}}{ }^{(i+1)} \boldsymbol{y}_{L}, \\
& \text { and/or } \quad{ }^{i} \tilde{\boldsymbol{p}}_{j R}={ }^{i} \boldsymbol{T}_{i+1}{ }^{i+1} \boldsymbol{T}_{(i+1)^{\prime}}{ }^{(i+1)^{\prime}} \tilde{y}_{R} \text {, } \\
& \text { where } \quad i^{\prime} \boldsymbol{T}_{i+1}=\boldsymbol{V}_{i} \text {, } \\
& { }^{i+1} \boldsymbol{T}_{(i+1)^{\prime}}=\boldsymbol{Q}_{i+1}, \\
& { }^{i} \tilde{p}_{j L} \equiv{ }^{w} \boldsymbol{T}_{i^{\prime}}\left(\boldsymbol{q}_{j}\right)^{-1}{ }^{w} \tilde{\boldsymbol{p}}_{j L}, \\
& { }^{i^{\prime}} \tilde{p}_{j R} \equiv{ }^{w} \boldsymbol{T}_{i^{\prime}}\left(\boldsymbol{q}_{j}\right)^{-1}{ }^{w} \tilde{p}_{j R}, \\
& { }^{(i+1)^{\prime}} \tilde{y}_{L} \equiv{ }^{(i+1)^{\prime}} \boldsymbol{T}_{6 L}\left(\boldsymbol{q}_{j}\right) \tilde{\boldsymbol{y}}_{L}, \\
& { }^{(i+1)^{\prime}} \tilde{y}_{R} \equiv{ }^{(i+1)^{\prime}} \boldsymbol{T}_{6 R}\left(\boldsymbol{q}_{j}\right) \tilde{y}_{R} \text {. }
\end{aligned}
$$

Note that if the $i$ th link is a common links, i.e., a link between the world reference frame and joint 4 , then both of the above two equations are valid; otherwise, only one of the equations (11) and (12) is valid.

From equations (11) and (12), we have

$$
\begin{array}{ll} 
& \boldsymbol{V}_{i}^{-1}{ }^{i} \tilde{p}_{j L}=\boldsymbol{Q}_{i+1}{ }^{(i+1)^{\prime}} \tilde{\boldsymbol{y}}_{L}, \\
\text { and/or } & \boldsymbol{V}_{i}^{-1}{ }^{i^{\prime}} \tilde{\boldsymbol{p}}_{j R}=\boldsymbol{Q}_{i+1}{ }^{(i+1)^{\prime}} \tilde{\boldsymbol{y}}_{\boldsymbol{R}} .
\end{array}
$$

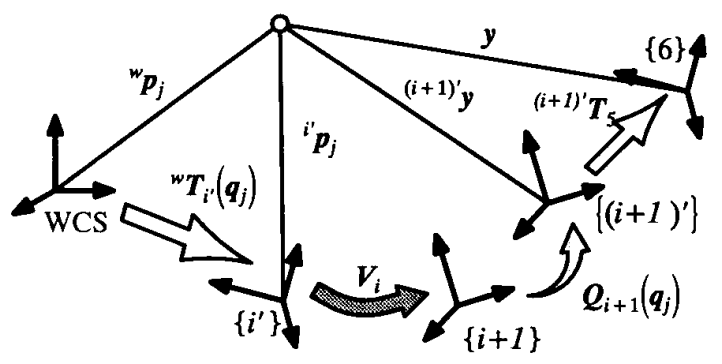

Fig. 4. Relation between 3D coordinates of a calibration point at the $\mathrm{jth}$ configuration w.r.t. different reference frames
If link $i$ is shared by both the left and right kinematic chains, then we take the average of equations (15) and (16), which yields

$$
V_{i}^{-1} i^{\prime} \tilde{p}_{j L R}=\boldsymbol{Q}_{i+1}{ }^{(i+1)^{\prime}} \tilde{y}_{L R},
$$

where

$$
{ }^{i} \tilde{\boldsymbol{p}}_{j L R}=\left({ }^{i} \tilde{\boldsymbol{p}}_{j L}+{ }^{\prime} \tilde{\boldsymbol{p}}_{j R}\right) / 2
$$

and $\quad{ }^{(i+1)^{\prime}} \tilde{y}_{L R}=\left({ }^{(i+1)^{\prime}} \tilde{y}_{L}+{ }^{(i+1)^{\prime}} \tilde{y}_{R}\right) / 2$.

$$
\text { Let }\left\{\begin{aligned}
i^{\prime} \tilde{\boldsymbol{p}}_{j}={ }^{\prime} \tilde{\boldsymbol{p}}_{j L R}, \quad{ }^{(i+1)^{\prime}} \tilde{\boldsymbol{y}}={ }^{(i+1)^{\prime}} \tilde{\boldsymbol{y}}_{L R}, \\
\\
\quad \text { if link } i \text { is shared by the left and right } \\
\quad \text { kinematic chains } \\
{ }^{\prime} \tilde{\boldsymbol{p}}_{j}={ }^{\prime} \tilde{\boldsymbol{p}}_{j L}, \quad{ }^{(i+1)^{\prime}} \tilde{\boldsymbol{y}}={ }^{(i+1)^{\prime}} \tilde{\boldsymbol{y}}_{L}, \\
\quad \text { if link } i \text { belongs to the left branch only } \\
i^{\prime} \tilde{\boldsymbol{p}}_{j}={ }^{\prime} \tilde{\boldsymbol{p}}_{j R}, \quad{ }^{(i+1)^{\prime}} \tilde{\boldsymbol{y}}={ }^{(i+1) '} \tilde{\boldsymbol{y}}_{\boldsymbol{R}}, \\
\text { if link } i \text { belongs to the right branch only }
\end{aligned}\right.
$$

Then, equations (15), (16) and (17) can be unified as the following

$$
V_{i}^{-1}{ }^{\prime} \tilde{p}_{j}=\boldsymbol{Q}_{i+1}{ }^{(i+1)} \tilde{\boldsymbol{y}}
$$

The unknown parameters to be estimated are the link matrix, $\boldsymbol{V}_{i}$, and the vector, ${ }^{(i+1)^{\prime}} \boldsymbol{y}$. For the case of IIS-Head, our goal is to estimate the kinematic parameters: $V_{0}, V_{1}, V_{2}, V_{3}, V_{4 L}$ and $V_{4 R}$, using equation (18). In this case, we can set $V_{5 L}=I$ and $V_{5 R}=\boldsymbol{I}$.

\section{New method for the kinematic parameter identification}

Using equation (18), our new method will calibrate the $i$ th link matrix by moving the $(i+I)$ st joint (joints $1,2, \ldots, i$ can be moved too). For convenience, let $R_{V_{i}}, R_{i}$ and $\operatorname{Rot}_{z}(\theta)$ be the $3 \times 3$ rotation matrix of $V_{i}, R_{i}$ and $\operatorname{Rot}_{z}(\theta)$, respectively, and let $t_{V_{i}}$ be the $3 \times 1$ translation vector of matrix $V_{i}$, and $l_{i}=\left[\begin{array}{lll}l_{i, x} & l_{i, y} & l_{i, z}\end{array}\right]^{T}$. Using equation (6), we have

$$
\begin{gathered}
R_{v_{i}}=R_{i} \operatorname{Rot}_{z}\left(\beta_{i}\right), \\
t_{V_{i}}=R_{V_{i}} l_{i},
\end{gathered}
$$

and

$$
V_{i}=\boldsymbol{R}_{V_{i}} \operatorname{Trans}\left(l_{i}\right) \text {, }
$$

which will be used in the following derivation.

\section{III.1 Kinematic parameter identification for a prismatic joint}

The redundant parameters and the unknowns for a prismatic joint are listed below for clarity of our derivation:

i). Four given redundant parameters (typically set to zero if not pre-specified for specific reason): $\beta_{i}$ and $\boldsymbol{l}_{i}$.

ii). The unknowns: $\boldsymbol{R}_{i},{ }^{(i+1)} \dot{\boldsymbol{y}}$ and the sign parameter, $s_{i}$.

Consider equation (18). For a prismatic joint, we have

$$
V_{i}^{-1}{ }^{i} \tilde{p}_{j}=\operatorname{Trans}\left(\left[\begin{array}{lll}
0 & 0 & q_{(i+1) j}
\end{array}\right]^{T}\right){ }^{(i+1)^{\prime}} \tilde{y},
$$

or, using (21),

$$
R_{V_{i}}^{T}{ }^{i} p_{j}-l_{i}={ }^{(i+1)^{\prime}} y+\left[\begin{array}{lll}
0 & 0 & q_{(i+1) j}
\end{array}\right]^{T} .
$$

Notice that the value of ${ }^{i} \boldsymbol{p}_{j}$ depend on the movements of 
joints $1, \ldots, i$ and can be computed using equations (13) and/ or (14).

Multiplying $R_{V_{i}}$ to both sides of equation (23) and noticing that

$$
R_{V_{i}}\left[\begin{array}{c}
0 \\
0 \\
q_{(i+1) j}
\end{array}\right]=R_{i} \operatorname{Rot}_{z}\left(\beta_{i}\right)\left[\begin{array}{c}
0 \\
0 \\
q_{(i+1) j}
\end{array}\right]=q_{(i+1) j} b_{i}
$$

where $b_{i}=\left[\begin{array}{lll}b_{i, x} & b_{i, y} & b_{i, z}\end{array}\right]^{T}$ is the third column vector of the rotation matrix $R_{i}$, we have

$$
{ }^{i} \boldsymbol{p}_{j}=\boldsymbol{a}+q_{(i+1) j} \boldsymbol{b}_{i},
$$

where $a \equiv R_{V_{i}}\left(\boldsymbol{l}_{i}+{ }^{(i+1)^{\prime}} \boldsymbol{y}\right)$ is independent of $j$. It is obvious that $l_{i}$ is redundant, since $l_{i}$ and ${ }^{(i+1)^{\prime}} y$ can not be independently estimated. Therefore, $\boldsymbol{l}_{i}$ can be set to zero if it is not pre-specified for specific reason.

Suppose there are $M$ measurements from $M$ robot configurations, i.e., ${ }^{\prime} \boldsymbol{p}_{j}$ and $q_{(i+1) j}, j=1,2, \ldots, M$. The kinematic parameter $\boldsymbol{b}_{i}$ can be estimated by minimizing the following error

$$
\varepsilon \equiv \sum_{j=1}^{M}\left\|i^{i} \boldsymbol{p}_{j}-\boldsymbol{a}-q_{(i+1) j} \boldsymbol{b}_{i}\right\|^{2},
$$

subject to $\boldsymbol{b}_{i}^{T} \boldsymbol{b}_{i}=1$.

The solution is (refer to [9] for the proof)

$$
\boldsymbol{b}_{i}=\sum_{j=1}^{M}\left(\Delta p_{j} \Delta q_{(i+1) j}\right) /\left\|\sum_{j=1}^{M}\left(\Delta p_{j} \Delta q_{(i+1) j}\right)\right\| \text {, }
$$

where

$$
\Delta \boldsymbol{p}_{j} \equiv{ }^{i} \boldsymbol{p}_{j}-{ }^{i} \overline{\boldsymbol{p}},
$$$$
\Delta q_{(i+1) j} \equiv q_{(i+1) j}-\bar{q}_{(i+1)} \text {, }
$$

and

$$
{ }^{\prime} \overline{\boldsymbol{p}}=\frac{1}{M} \sum_{j=1}^{M} i^{\prime} \boldsymbol{p}_{j}
$$

$$
\bar{q}_{(i+1)}=\frac{1}{M} \sum_{j=1}^{M} q_{(i+1) j}
$$

Notice that if the third component of $\boldsymbol{b}_{i}$ is negative, in order to be consistent with the CPC convention, we should change the sign of $b_{i}$ and let $s_{i}=-1$; otherwise, let $s_{i}=+1$. Once the unit vector $\boldsymbol{b}_{i}$ is obtained, the rotation matrix, $\boldsymbol{R}_{i}$, can be computed with equation (7). Finally, ${ }^{(i+1)^{\prime}} \boldsymbol{y}$ can be solved by using equation (23). However, we do not have to calculate ${ }^{(i+1)} y$ if it is not of interest.

\section{III.2 Kinematic parameter identification for a revolute joint}

The redundant parameters and the unknowns for a revolute joint are listed below for clarity of the derivation:

(1). Two given redundant parameters (typically set to zero if not pre-specified for specific reason): $\beta_{i}$ and the $\mathrm{z}-$ component of $\boldsymbol{l}_{i}$.

(2). The unknowns: $\boldsymbol{R}_{i}$, the sign parameter, $s_{i},{ }^{(i+1)} \boldsymbol{y}$, and the first two components of $\boldsymbol{l}_{\boldsymbol{i}}$.

For a revolute joint, the calibration equation can be derived from (18) that

$$
R_{V_{i}}^{T}{ }^{i} \boldsymbol{p}_{j}-\boldsymbol{l}_{i}=\operatorname{Rot}_{z}\left(q_{(i+1) j}\right)^{(i+1)^{\prime}} \boldsymbol{y},
$$

by using equation (21). For convenience, we decompose ${ }^{(i+1)^{\prime}} \boldsymbol{y}$ into two portions as follows. Let ${ }^{(i+1)^{\prime}} \boldsymbol{y}=\left[\begin{array}{lll}y_{1} & y_{2} & y_{3}\end{array}\right]^{T}$, and

$$
{ }^{(i+1)^{\prime}} \boldsymbol{y}=\boldsymbol{y}_{a}+\boldsymbol{y}_{b}
$$

where $\boldsymbol{y}_{a}=\left[\begin{array}{lll}y_{1} & y_{2} & 0\end{array}\right]^{T}$ and $\boldsymbol{y}_{b}=\left[\begin{array}{lll}0 & 0 & y_{3}\end{array}\right]^{T}$. Note that for any $y_{1}$ and $y_{2}, y_{1}^{2}+y_{2}^{2} \neq 0$, there exist a scalar $\varrho$ and a rotation angle $\omega$ such that

$$
y_{a}=\varrho \operatorname{Rot}_{z}(\omega) e_{1},
$$

where $e_{1}=\left[\begin{array}{lll}1 & 0 & 0\end{array}\right]^{T}, \varrho=\sqrt{y_{1}^{2}+y_{2}^{2}}, \omega$ is the angle between the vector $y_{a}$ and the $x$-axis. Substituting equations (33) and (34) into equation (32), we have (by noting that $\left.y_{b}=\operatorname{Rot}_{z}(\cdot) y_{b}\right)$

$$
R \boldsymbol{u}_{i}+\boldsymbol{t}=\varrho \boldsymbol{v}_{j},
$$

where $R \equiv \operatorname{Rot}_{z}\left(-\beta_{i}-\omega\right) R_{i}^{T}, u_{j} \equiv{ }^{i} p_{j}$, $t \equiv-\operatorname{Rot}_{z}(-\omega) l_{i}-y_{b}$ and $v_{j} \equiv \operatorname{Rot}_{z}\left(q_{(i+1) j}\right) e_{1}$.

$R, t$ and $\varrho$ can be solved by minimizing the following error using the least square method.

$$
e \equiv \sum_{j=1}^{M}\left\|R u_{j}+t-\varrho v_{j}\right\|^{2}
$$

The procedures for calculating a closed-form solution to equation (36) are listed in the following (refer to [9] for more details).

Step 1: Compute $\overline{\boldsymbol{u}} \equiv \frac{1}{N} \sum_{j=1}^{M} \boldsymbol{u}_{j}, \overline{\boldsymbol{v}} \equiv \frac{1}{N} \sum_{j=1}^{M} \boldsymbol{v}_{j}, \underline{\boldsymbol{u}}_{j}=\boldsymbol{u}_{j}-\overline{\boldsymbol{u}}$ and $\underline{\boldsymbol{v}}_{j}=\boldsymbol{v}_{j}-\overline{\boldsymbol{v}}$.

Step 2: Let $\boldsymbol{A} \equiv\left[\begin{array}{lllll}\underline{v}_{1} & \cdots & \underline{v}_{j} & \ldots & \underline{v}_{M}\end{array}\right]$ and $\boldsymbol{B} \equiv\left[\begin{array}{lllll}\underline{\boldsymbol{u}}_{1} & \cdots & \underline{\boldsymbol{u}}_{j} & \cdots & \underline{\boldsymbol{u}}_{M}\end{array}\right]$.

Step 3: Compute the matrix $\boldsymbol{C} \equiv \boldsymbol{B} \boldsymbol{A}^{T}$.

Step 4: Compute the singular value decomposition

$$
C=U\left[\begin{array}{ccc}
s_{1} & 0 & 0 \\
0 & s_{2} & 0 \\
0 & 0 & s_{3}
\end{array}\right] V^{T} \text {, where } s_{1} \geq s_{2} \geq s_{3} \geq 0 .
$$

Step 5: If $\operatorname{det}\left(\boldsymbol{V} \boldsymbol{U}^{T}\right)=+1$, then compute $\hat{\boldsymbol{R}}=\boldsymbol{V} \boldsymbol{U}^{T}$,

$$
\text { otherwise, let } \hat{R}=V\left[\begin{array}{ccc}
1 & 0 & 0 \\
0 & 1 & 0 \\
0 & 0 & -1
\end{array}\right] U^{T} \text {. }
$$

Step 6: Compute $\varrho=\left(\sum_{j=1}^{M} \underline{u}_{j}^{T} R \underline{v}_{j}\right)\left(\sum_{j=1}^{M} \underline{v}_{j}^{T} \underline{v}_{j}\right)^{-1}$.

Step 7: Compute $\boldsymbol{t}=\varrho \overline{\boldsymbol{v}}-\boldsymbol{R} \overline{\boldsymbol{u}}$.

We now show how to compute the kinematic parameters, $R_{i}$ and $\boldsymbol{l}_{i}$, from $R$ and $t$. Remember that $R=\operatorname{Rot}_{z}\left(-\beta_{i}-\omega\right) R_{i}^{T}$, as defined following equation (35). It is obvious that the third rows of $R$ and $R_{i}^{T}$, i.e., $b_{i}^{T}$, should be the same. Therefore, $b_{i}^{T}$ can be obtained from the third row of the matrix $R$. Notice that if the third component 


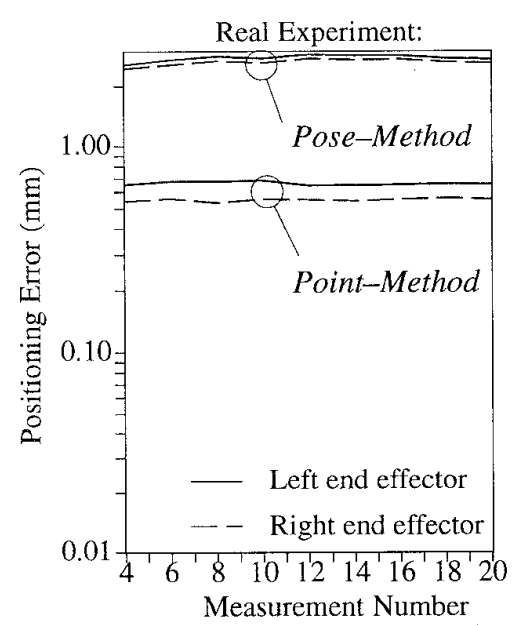

Fig. 5. The positioning error of two end effectors versus the measurement number (19 test points)

of $\boldsymbol{b}_{i}$ is negative, in order to be consistent with the CPC convention, we should change the sign of $\boldsymbol{b}_{i}$ and let $s_{i}=-1$; otherwise, let $s_{i}=+1$. From equations (6) and (7), we can compute $R_{V_{i}}$ from $\boldsymbol{b}_{i}$ and $\beta_{i}$. Then we can compute $\operatorname{Rot}_{z}(\omega)$ using the following relation:

$$
\operatorname{Rot}_{z}(\omega)=R_{V_{i}}^{T} R^{T}
$$

Remember that $t=-\operatorname{Rot}_{z}(-\omega) l_{i}-y_{b} \quad$ and $\boldsymbol{y}_{b}=\left[\begin{array}{lll}0 & 0 & y_{3}\end{array}\right]^{T}$, hence

$$
l_{i}+y_{b}=-\operatorname{Rot}_{z}(\omega) \boldsymbol{t},
$$

where the $\mathrm{z}$-component of $\boldsymbol{l}_{i}$ is a given redundant parameter, and $\boldsymbol{y}_{b}$ should be determined to make the above three equations of three unknowns consistent.

\section{Experiments}

To evaluate the accuracy of robot calibration, we use the positioning error defined below as the error measure. The positioning error is defined as the Euclidian distance between the measured position of the end-effector (or more precisely, of the single calibration point) and its predicted position using the identified CPC kinematic model. The 3D measurement system we use is a stereo vision system with baseline of 0.5 meter, which provides the accuracy of $0.4 \mathrm{~mm}$ in $\mathrm{z}$-direction and $0.2 \mathrm{~mm}$ in $\mathrm{x}$-or $\mathrm{y}$-directions if the object distance is approximately 1.5 meters and the deviation of the 2D observation error is 0.1 pixel. Each small calibration plate attached to the camera mount has nine white disks. For each measurements, the estimated 3D coordinates of the nine disks are averaged to obtain an accurate estimate of the endeffector's 3D position, which has approximately 0.05 millimeter standard deviation in each direction.

For comparison, we have also implemented a kinematic calibration method using pose measurements[8]. To obtain the pose measurements, a fixed coordinate system called the

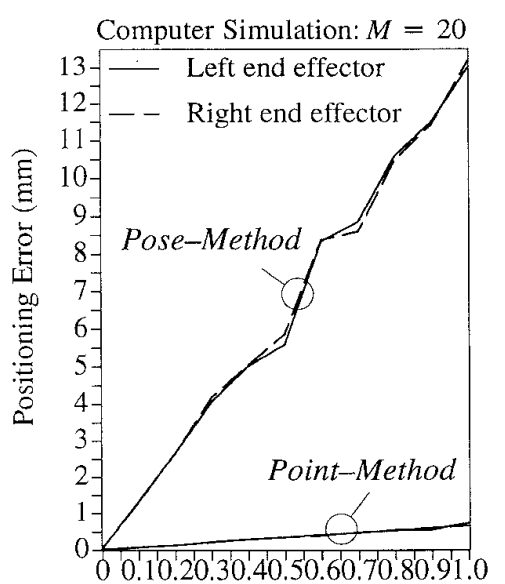

Std. Dev. of 2D Observation Error (in pixel)

Fig. 7 The positioning error of two

end effectors versus the measurement noise. left (right) calibration plate coordinate system (LCPCS, RCPCS) is defined on the left (right) calibration plate. The coordinates of the centroid of each disk on the calibration plate with respect to LCPCS or RCPCS are known a priori. The pose measurements are obtained by applying Arun's Algorithm[2] to the 3D coordinates of the nine disks measured in the LCPCS (RCPCS) and the WCS, respectively. For convenience, the method using pose measurements [8] will be referred to as the Pose-Method, and our new method will be referred to as the Point-Method.

In the first experiment (real experiment), twenty measurements are taken for each joint. Also, for testing the calibration accuracy we have taken extra nineteen measurements with arbitrary configurations. We then choose $M$ from the twenty measurements to calibrate the robot and use all of the nineteen testing measurements to test the estimated parameters. The results are shown in Fig. 5 for $M=4,6, \ldots, 20$. One purpose of this experiment is to determine a proper $M$ such that the position error is less than $1 \mathrm{~mm}$. Fig. 5 shows that the Point-Method can achieve higher accuracy than the PoseMethod. Notice that Fig. 5 does not show the potential that the position error decreases when $M$ increases. We believe it is because the non-geometric error dominates and the random noise induced error is relatively negligible. However, whatever $M$ is, the error is less than 1 millimeter. We use $M=$ 20 in the following experiments.

In the second experiment, we run simulations using the kinematic parameters obtained from the previous real experiment. We assume that the 3D stereo measurement noise is due to the 2D image observation noise of 0.1 pixel standard deviation. Each data points in Fig. 6 is the average of fifty random trials. Notice that in the real experiments, the test data also contain measurement errors. Also, the joints of the IIS-Head are not ideal at all. Therefore, the real experimental results (in Fig. 5) have larger position error than the simulation ones (Fig. 6). 
The last experiment is to test the robustness of the calibration algorithm. The 2D observation error is assumed to be a normal random noise with zero mean and deviation $\sigma_{2 D}$. Fig. 7 shows the results from computer simulations with each point is obtained from the average of fifty random trials, for $\sigma_{2 D}=0.0-1.0$ pixel. The number of measurements for each joint is set to 20 as in the real experiment. This experiment shows that the proposed method is robust against the observation noise.

\section{Conclusions}

Active vision is attracting more and more research interest in the field of computer vision. Being able to acquire information actively, the active vision system has more potential applications than a passive one has. To control the positions and orientations of the cameras, the kinematic parameters of the active vision mechanism have to be known. Unfortunately, the exact kinematic parameters are usually unknown. In this paper we have solved the kinematic parameters identification problem for the IIS-Head based on the CPC kinematic model. Three main features of our new method are described below:

1). With our new method, the kinematic parameters estimation problem is decomposed into many subproblems of single joint axis. Hence, the complexity is reduced and an easier implementation is derived. Also, this decomposition leads to a general solution for any robot with arbitrary combination of prismatic and revolute joints. Notice that this does not mean that only one joint can be moved at a time during the data collection phase. Instead, all the calibrated joints can be moved to gather more information.

2). It is easier to obtain an accurate point measurement than an accurate pose measurement. Moreover, the calibration object can be of smaller size when using point measurements, instead of pose measurements, which allows larger range of joint movement in calibration and leads to more accurate and robust estimations of kinematic parameters. Our method, unlike others using single calibration point described in [6], can provide the transformations from tool to end-effector and from world to base (refer to [9]).

3 ). With our method, each joint is calibrated in the order of from the base to the end-effectors. Therefore, it is very suitable for kinematic calibration of robots having multiple endeffectors. On the contrary, if we calibrate a multiple end-effector robot from the end-effectors to the base as those methods described in [8] and [14], then at the link having two branching kinematic chains, we need to estimate an additional transformation matrix for unifying the coordinates systems from different end-effectors (as in [7]).

The proposed closed-form solution method has been applied to solve the kinematic calibration problem of a branched kinematic chain having two end-effectors. The proposed method has been tested and the results show that our method of using point measurements can achieve much higher accuracy than that of using pose measurements.

\section{Acknowledgement}

This research is supported in part by the National Science Council, Taiwan, ROC, under grant NSC-83-0408-E-001-004 .

\section{References}

[1] J. Aloimonos and A. Badyopadhyay, "Active Vision," IEEE Proceedings of the first Int. Conf. on Computer Vision, pp. 35-54, June 1987.

[2] K. S. Arun, T.S. Huang, and S.D. Blostein, "Least-Square Fitting of Two 3-D Point Sets," IEEE Trans. on Pattern Analysis and Machine Intelligence, vol. PAMI-9, NO. 5, pp. 698-700, 1987.

[3] S. Hayati, K. Tso and G. Roston, "Robot Geometry Calibration," Proceedings of the Int. Conf. on Robotics and Automation, pp. 947-951, 1988.

[4] R.K. Lenz, R.Y. Tsai, "Calibrating a Cartesian Robot with Eyeon-Hand Configuration Independent of Eye-to-Hand Relationship," IEEE Trans, on Pattern Analysis and Machine Intelligence, vol. 11, no. 9, pp. 916-928, Sep. 1989.

[5] B.W. Mooring, T. J. Pack, "Calibration Procedure for an Industrial Robot," Proceedings of the Int. Conf. on Robotics and Automation, pp. 786-791, 1988.

[6] B.W. Mooring, Z.S. Roth and M.R. Driels, Fundamentals of Manipulator Calibration, A Wiley-Interscience Publication, John Wiley \& Sons, Inc., 1991.

[7] S.W. Shih, J.S. Jin, K.H. Wei, Y.P. Hung, "Kinematic Calibration of a Binocular Head Using Stereo Vision with the Complete and Parametrically Continuous Model," SPIE Proceedings, Intelligent Robots and Computer Vision XI, vol. 1825, pp. 643-657, 1992.

[8] S.W. Shih, Y.P. Hung and W.S. Lin, "Comments on A Linear Solution to Kinematic Parameter identification of Robot Manipulator," to appear in IEEE Trans. Robotics and Automation.

[9] S.W. Shih, Y.P. Hung and W.S. Lin, "Kinematic Parameter Identification of an Binocular Head Using Stereo Vision," Technical Report TR-94-006, Institute of Information Science, Academia Sinica, Nankang, Taipei, Taiwan, 1994.

[10] H.W. Stone, Kinematic Modeling, Identification, and Control of Robotic Manipulators. Norwell, MA: Kluwer Academic, 1987.

[11] M.E. Sklar, "Metrology and Calibration Techniques for the Performance Enhancement of Industrial Robots," Ph.D. Dissertation, the University of Texas at Austin, 1988.

[12] G.S. Young, T.H. Hong, M. Herman and J.C.S. Yang, "Kinematic Calibration of an Active Camera System," IEEE Proceedings of the Int. Conf. on Computer Vision Pattern Recognition, pp. 748-751, 1992.

[13] H. Zhuang, Z.S. Roth, F. Hamano, "A Complete and Parametrically Continuous Kinematic Model for Robot Manipulators," IEEE Trans. on Robotics and Automation, vol. 8, no. 4, pp. 451-463, Aug. 1992.

[14] H. Zhuang, Z.S. Roth, "A Linear Solution to the Kinematic Parameter Identification of Robot Manipulators," IEEE Trans. on Robotics and Automation, Vol. 9, No. 2, pp. 174-185, April 1993. 